\title{
The Complexity of Propositional Modal Theories and the Complexity of Consistency of Propositional Modal Theories*
}

\author{
Cheng-Chia Chen and I-Peng Lin \\ Department of Computer Science and Information Engineering, \\ National Taiwan University, Taipei, Taiwan, R.O.C.

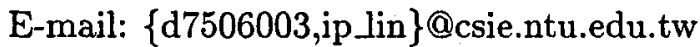

February 16, 1994

Keyword: computational complexity, modal logic, deducibility, consistency, provability, satisfiability.

\begin{abstract}
This paper is concerned with the computational complexity of the following problems for various modal logics $L$ : (1). The $L$-deducibility problem: given a finite set of formulas $S$ and a formula $A$, determine if $A$ is in the modal theory $T H_{L}(S)$ formed with all theorems of the modal logic $L$ as logical axioms and with all members of $S$ as proper axioms. (2). The $L$-consistency problem: given a finite set of formulas $S$, determine if the theory $T H_{L}(S)$ is consistent.

Table 1 is a comparison of complexity results of these two problems and the corresponding provability and satisfiability problems for modal logics K, T, B, S4, KD45 and S5. The complexity results of the deducibility problem for extensions of $\mathrm{K} 4$ are a direct consequence of a modal deduction theorem for $\mathrm{K} 4$ (cf. $[17,15])$. The NP-completeness of the S4-consistency problem is due to Tiomkin and Kaminski [15].

The main contribution of this paper is that we can show that the deducibility problem and the consistency problem for any modal logic between $\mathrm{K}$ and $\mathrm{B}$ are EXPTIMEhard; in particular, for $\mathrm{K}, \mathrm{T}$ and $\mathrm{B}$, both problems are EXPTIME-complete.
\end{abstract}

*This research was supported by the National Science Council of ROC under contract number NSC820408-E-002-428. 
Table 1: The complexity of various decision problems for some normal modal logics. Note: all problems are complete for the corresponding complex classes.

\begin{tabular}{|l|l|l|l|l|l|}
\hline Logic(L) & $\mathrm{K}$ & $\mathrm{T}$ & $\mathrm{B}$ & $\mathrm{S} 4$ & $\mathrm{KD} 45, \mathrm{~S} 5$ \\
\hline \hline L-provability & PSPACE & PSPACE & PSPACE & PSPACE & Co-NP \\
\hline L-satisfiability & PSPACE & PSPACE & PSPACE & PSPACE & NP \\
\hline L-deducibility & EXPTIME & EXPTIME & EXPTIME & PSPACE & Co-NP \\
\hline L-consistency & EXPTIME & EXPTIME & EXPTIME & NP & NP \\
\hline
\end{tabular}

\section{Introduction}

In the study of logic, there are two kinds of relations that are usually discussed. One is a unary relation consisting of formulas derivable from the logical axioms and inference rules of a logic and is called the provability relation; the other is a binary relation, which we call the deducibility relation and is concerned with whether a given formula can be derived from the logic with an additional set of formulas given as proper axioms. The provability relation is always a subrelation of the deducibility relation with the given set of proper axioms restricted to empty.

For classical logic, since the deduction theorem holds, if the set of proper axioms is restricted to finite sets, the deducibility relation can be reduced to the provability relation. As a result both relations are inter-reducible and any complexity results about either one apply to the other too.

For modal logic, however, there are at least two deducibility relations that have been defined in the literature [7] depending on whether we admit the rule of necessitation(NEC): "If $S \vdash A$, then $S \vdash \square A$ ". For the deducibility relation without the rule NEC, the deduction theorem also holds. Hence its decision problem is reducible to the corresponding provability relation. Consequently, any complexity result about this relation can resort to the better known result of the corresponding provability relation. For this reason, we need only pay our attention to the deducibility relation admitting the rule NEC.

Under the deducibilty relation discussed, we can also define the notion of cosistency. We say that a set of formulas is consistent for a modal logic L (or simply L-consistent) if $\perp$ (falsity) is not derivable from the logic with the set of formulas given as proper axioms.

In this paper we are concerned with the complexity of the deducibility relations and the corresponding consistency relations for some modal logics. We restrict the given set of proper axioms in both relations to finite sets.

In the study of modal logic, traditional logicians seem more interested in the provability relation and its corresponding satisfiability relation; in contrast, the deducibility relation receives less attention. Nevertheless, in the application of modal logics to artificial intelligence, the deducibility relation has been found to be fundamental for nonmonotonic reasoning $[12,15,14]$. In fact, our study of the complexity of these 
relations is motivated by Moore's autoepistemic logic (AEL)[13] and McDermott's Nonmonotonic modal logic (NML) [12], each suggesting a fixed point semantics and the corresponding fixed point operator being defined in terms of the deducibility relations in some modal logics.

Table 1 is a comparison of the complexities of these two problems and the corresponding provability and satisfiability problems for modal logics K, T, B, S4, KD45 and S5. The complexity results of the deducibility problem for extensions of $\mathrm{K} 4$ are a direct consequence of a modal deduction theorem for $\mathrm{K} 4$ ( $\mathrm{cf}[17,15])$. The NP-completeness of the S4-consistency problem is due to Tiomkin and Kaminski [15].

The main results of this paper is that we can show that the deducibility problem and the consistency problem for any modal logic between $K$ and $B$ are EXPTIME-hard; in particular, for $K, T$ and $B$, both problems are EXPTIME-complete.

The rest of this paper is organized as follows. In Section 2 we define the deducibility and the consistency relations and their semantics. Section 3 summarizes all complexity results. Section 4 presents the upper bound result by showing that the deducibility relation for $\mathrm{K}, \mathrm{T}$ and $\mathrm{B}$ is in EXPTIME. Finally, in Section 5 we presents the lower bound result by showing that the consistency relation for any normal logic between $K$ and $B$ is EXPTIME-hard.

\section{Preliminaries}

All modal logics considered in the paper share a common language whose alphabet $\Gamma$ includes : (1). a denumerable set $P V$ of propositional variables: $p, q, r \cdots$ and (2). the five symbols: (, ), $\neg, \wedge, \square$. The set of modal formulas $M F$ is defined to be the least set of words over $\Gamma$ including $P V$ such that if $A$ and $B$ are members of $M F$, then so are $(A \wedge B), \neg A$ and $\square A$. The other connectives such as $\vee$ (or), $\supset$ (implication), $\diamond$ (possibility), $\perp$ (falsity) as treated as abbreviations in the standard way.

By a (modal) logic $L$ we mean any subset of $M F$ that contains all tautologies and is closed under modus ponens (MP) and uniform substitution (US). So the classical propositional logic (in $M F$ ) is the smallest modal logic, while the set of all modal formulas is the largest modal logic.

We say that a modal logic $L$ is normal if it contains the formula $K: \square(p \supset q) \supset$ $(\square p \supset \square q$ ) and is closed under necessitation(NEC): $\square A \in L$ whenever $A \in L$. The smallest normal modal logic is called $K$.

There are famous normal modal logics that can be defined as smallest normal logics containing parts of the following formulas:

D: $\square p \supset \diamond p$.

T: $\square p \supset p$.

B: $p \supset \square \diamond p$.

4: $\square p \supset \square \square p$.

5: $\square p \supset \diamond \square p$. 
As adopted by Chellas[2], we use $K a_{1} \cdots a_{n}$, where $n \geq 0$ and each $a_{i} \in\{D, T, B, 4,5\}$, to denote the smallest normal modal logic containing all logical axioms $a_{i}$ 's. According to such convention, the five famous normal modal logics more usually called $D$, $T, B, S 4$ and $S 5$ are in fact equal to $K D, K T, K T B, K T 4$ and $K T 45$, respectively. In what follows let $\mathcal{M}$ denote the set of logics $\left\{L \mid L=K a_{1} \cdots a_{n}, n \geq 0\right.$ and each $\left.a_{i} \in\{D, T, B, 4,5\}\right\}$.

Each member of a modal logic is called a theorem of the logic. We use the relational notation $\vdash_{L} A$ to stand for the fact that $A \in L$. In [11], $\vdash_{L}$ is called the provability relation for $L$ (or simply the $L$-provability relation). For any logics $L_{1}, L_{2}$, we say $L_{2}$ is an extension of $L_{1}$ (and $L_{1}$ is a sublogic of $L_{2}$ ) iff $L_{1}$ is a subset of $L_{2}$. We say another $\operatorname{logic} L$ is between $L_{1}$ and $L_{2}$ if $L_{1} \subseteq L \subseteq L_{2}$. Therefore, the normal logics $D, T$, and $K 4$ are between $K$ and $S 4 ; D, T, B$ and $S 4$ are all extensions of $K$.

Let $L$ be any modal logic, the L-deducibility relation, with some abuse of terminology, also denoted $\vdash_{L}$, is defined to the the smallest subset of $2^{M F} \times M F$ closed under the following rules:

1. $S \vdash_{L} A$ whenever $A \in S$ or $A$ is a theorem of $L$ (Axioms).

2. $S \vdash_{L} B$ if $S \vdash_{L} A$ and $S \vdash_{L} A \supset B(\mathrm{MP})$.

3. $S \vdash_{L} \square A$ if $S \vdash_{L} A$ (NEC).

The $L$-theory of $S$, denoted $T H_{L}(S)$, is the set $\left\{A \in M F \mid S \vdash_{L} A\right\}$. If $S \vdash_{L} A$, we say $A$ is deducible from $S$ under $L$. Each formula in $S$ is called a proper axiom of the theory.

We say that a set of formulas $S$ is $L$-consistent if $\perp$ (falsity) $\notin T H_{L}(S)$.

\subsection{Semantics}

The semantics of many modal logics can be defined by Kripke model. A (Kripke) model $M$ is a triple $\langle W, R, h\rangle$ where

- $W$ is a nonempty set (of worlds),

- $R$ is a binary relation on $W$ called the accessibility relation of $M$; if $\left(w, w^{\prime}\right) \in R$, we say $w^{\prime}$ is accessible from $w$. The pair $(W, R)$ is called the frame of $\mathrm{M}$.

- $h \in W \rightarrow 2^{P V}$ is the valuation function, which assigns to each world $w$ in $W$ a subset $h(w)$ of $P V$ with the intention that $p$ is true at world $w$ iff $p \in h(w)$.

Given any Kripke model $M=\langle W, R, h\rangle$, any world $w \in W$, and any formula $A \in M F$, the truth definition of $A$ at $w$ of $M$, denoted $M, w \models A$, is defined inductively as follows.

- $M, w \models p$ iff $p \in h(w)$

- $M, w \vDash \neg A$ iff $M, w \not A$

- $M, w \vDash A \wedge B$ iff $M, w \vDash A$ and $M, w \vDash B$.

- $M, w \vDash \square A$ iff for every $w^{\prime} \in W$ accessible from $w, M, w^{\prime} \vDash A$. 
We say $A$ is M-satisfiable if there is a world $w$ in $W$ such that $M, w \vDash A$ and say $A$ is M-valid, denoted $M \models A$, if $M, w \models A$ for every world $w$ in $W$.

We are particularly interested in Kripke models whose accessibility relations $R$ satisfy parts of the following conditions:

serial(D): for any $w \in W$, there is a $w^{\prime}$ in $W$ such that $w R w^{\prime}$.

reflexive( $\mathrm{T})$; for any $w \in W, w R w$.

symmetric(B): for any $w, w^{\prime} \in W$, if $w R w^{\prime}$ then $w^{\prime} R w$.

transitive(4): for any $w, w^{\prime}, w^{\prime \prime} \in W$, if $w R w^{\prime}$ and $w^{\prime} R w^{\prime \prime}$, then $w R w^{\prime \prime}$.

euclidean(5): for any $w, w^{\prime}, w^{\prime \prime} \in W$, if $w R w^{\prime}$ and $w R w^{\prime \prime}$, then $w^{\prime} R w^{\prime \prime}$.

With some abuse of terminology, we use $K r_{1} \cdots r_{n}$, where $(n \geq 0)$ and each $r_{i} \in\{D, T, 4,5, B, H\}$, to denote also the class of all kripke models whose accessibility relations satisfy all $r_{i}$ 's. Each member of the class $K r_{1} \cdots r_{n}$ of models is called a $K r_{1} \cdots r_{n}$-model. Analogous to modal logics, we also use T, D, B, S4 and S5 to denote the classes of KT, KD, KTB, KT4 and KT45 models, respectively.

Let $L$ be any class of models. We say that a formula $A$ is (locally) $L$-satisfiable if there exists an L-model $M$ and a world $w$ in $W$ such that $M, w \vDash A$; we say $A$ is globally $L$-satisfiable if there is an L-model in which $A$ is valid. We say that $A$ is $L$-valid, denoted $\models_{L} A$, if $A$ is valid in every $L$-model.

By treating every (finite) set of formula as an abbreviation of the conjunction of all its members, we extend the definitions of previously defined notions like satisfiability, validity, etc. to sets of formulas in the obvious way. So, for example, $M, w \models S$ iff $M, w \models A$ for every $A \in S$.

The following well-known proposition establishes the equivalence of the semantical validity relation and the syntactic provability relation for each modal logic given here.

Proposition 1 [2] Let $L$ be any logic in $\mathcal{M}$. Then for any modal formula $A, A$ is $L$-provable iff $A$ is $L$-valid.

For each normal logic $L \in \mathcal{M}$, Kripke's model can also supply suitable semantics for the L-deducibility relation and the L-consistency relation. Given a set of formula $S$ and a formula $A$, we say that $A$ is a global $L$-consequence of $S$ if for all $L$-models $M$, if every formula of $S$ is valid in $M$, then so is $A$. D. McDermott [12] has shown that for $L \in\{T, S 4, S 5\}$, the deducibility relation and the consequence relation are identical. The following proposition is a simple generalization of his result.

Proposition 2 Let $L$ be any logic in $\mathcal{M}$. Then for any set of formula $S$ and for any formula $A, S \vdash_{L} A$ iff $A$ is a global $L$-consequence of $S$.

The following is a corollary of Proposition 2.

Proposition 3 Let $L$ be any logic in $\mathcal{M}$. Then for any set of formula $S, S$ is $L$ consistent iff $S$ is globally $L$-satisfiable. 


\section{Complexity results}

We now begin to study the complexity of the deducibility relation and the consistency relation for the logics $\mathrm{K}, \mathrm{T}, \mathrm{B}, \mathrm{S} 4, \mathrm{KD} 45$ and $\mathrm{S} 5$. The results, together with the complexity of other related decision problems, are listed in Table 1 . The complexity results of the provability and the satisfiability problems for these logics can be found in $[11,8,3]$.

For all extensions of $\mathrm{K} 4$ we have the following modal deduction theorem.

Proposition $4[7,15,17]$ Let $L$ be any extension of $K 4$. Then $S \vdash_{L} A$ iff $\vdash_{L}(\wedge S \wedge$ $\bigwedge\{\square B \mid B \in S\}) \supset A$.

According to Proposition 4, for every extension of $K 4$, the deducibility relation and the unary provability relation are inter-reducible and hence are in the same complexity class. Since the provability relation for S4 is PSPACE-complete [11] and the provability relation for KD45 and S5 $[11,8]$ is CoNP-complete, we thus have the following corollary.

\section{Corollary 1 1. The S4-deducibility relation is PSPACE-complete.}

2. The KD45-deducibility relation and the S5-deducibility relation are CoNP-complete.

That the L-consistency relation for every modal logic L is NP-hard is rather obvious since every Boolean formula(modal formula without modality) is satisfiable for classical propositional logic if and only if it is L-consistent. Hence the consistency relations for KD45 and S5, like their corresponding satisfiability problems, are NP-complete.

We might guess that for $\mathrm{S} 4$ the consistency problem has the same complexity as the satisfiability problem. But in fact the guess is incorrect. The following result is due to Tiomkin and Kaminski [15].

Lemma 2 [15] Let $S$ be any set of modal formulas. Then $S$ is $S 4$-consistent iff $S$ is S5-consistent.

Corollary 3 The consistency relation for any of $S 4, K D 45$ and $S 5$ is NP-complete.

Compared with the satisfiability problem, for $\mathrm{S} 4$ the consistency relation becomes easier to decide. On the contrary, for all normal logics between $K$ and $B$, we will show in Section 5 that the consistency relation is EXPTIME-hard; hence for $K, T$ and $B$ the global consistency relation is harder than the satisfiability relation, which has been shown to be PSPACE-complete.

\section{The deducibility relations for $\mathrm{K}, \mathrm{T}$ and $\mathrm{B}$ are in EXPTIME}

In this section we show that the global deducibility relations (and the global consistency relations) for the three modal logics $K, T$ and $B$ are all in EXPTIME. The result in 
fact also holds for many other logics between $K$ and $B$, such as $D, K B, O M, O M^{+}$, $O B, O B^{+}[16]$ etc. For simplicity, we choose here only $K, T$ and $B$ as representatives.

To show that the deducibility relation is in EXPTIME, our strategy is to use the semantical notion and show that the global consequence relations for $K, T$ and $B$ can all be reduced to the unsatisfiability problem of propositional dynamic logic with converse (CPDL). Since the latter problem is known to be EXPTIME-complete [9], the deducibility relations thus are in EXPTIME as well. For an understanding of this section, we assume the readers are familiar with propositional dynamic logic with converse. Readers who are unfamiliar with CPDL are referred to Harel[9]

\section{Translations of modal formulas into CPDL formulas}

Let $L$ be any of K, T and B. Let $a$ be a fixed atomic program. Define the CPDL programs $p r_{L}$ as follows.

1. $p r_{K}=a$,

2. $p r_{T}=a \cup \mathrm{T}$ ?,

3. $p r_{B}=a \cup a^{-} \cup \top$ ? .

For each modal formula $A$, define the CPDL formula

$$
\operatorname{Tr}_{L}(A)=A\left(\square \leftarrow\left[p r_{L}\right]\right)
$$

where $A\left(\square \leftarrow\left[p \tau_{L}\right]\right)$ is the CPDL formula obtained from $A$ with every occurrence of the $\square$ connective in $A$ replaced by the modality " $\left[p r_{L}\right]$ ".

For each pair $(S, A)$ where $S$ is a finite set of modal formulas and $A$ is a modal formula, now define three CPDL formulas

1. $f_{K}(S, A)=\left(\left[a^{*}\right] \operatorname{Tr}_{K}(\bigwedge S)\right) \wedge \operatorname{Tr}_{K}(\neg A)$,

2. $f_{T}(S, A)=\left(\left[a^{*}\right] \operatorname{Tr}_{T}(\bigwedge S)\right) \wedge \operatorname{Tr}_{T}(\neg A)$,

3. $f_{B}(S, A)=\left(\left[\left(a \cup a^{-} \cup \mathrm{T} \text { ? }\right)^{*}\right] \operatorname{Tr}_{B}(\wedge S)\right) \wedge \operatorname{Tr}_{B}(\neg A)$.

Intuitively, the formula $f_{B}(S, A)$ say that there is a CPDL structure $M$ and there is a world w at which $A$ is false and at every world reachable from which $S$ is true.

Lemma 4 [3] Let $L$ is any of $K, T$ and $B, S$ a finite set of modal formulas and $A$ a modal formula. Then $A$ is not an $L$-consequence of $S$ iff $f_{L}(S, A)$ is satisfiable for $C P D L$.

It is easy to see that $f_{L}(S, A)$ can be obtained from $S$ and $\mathrm{A}$ in polynominal time; moreover, since the unsatisfiability problem for CPDL is known to be in EXPTIME [9]. We thus can have the following corollary.

Corollary 5 For $L \in\{K, T, B\}$, the $L$-deducibility relation and the $L$-consistency relation are in EXPTIME. 


\section{EXPTIME-hardness of the consistency rela- tions for logics between $K$ and $B$}

We now turn to the derivation of lower bound results.

Theorem 1 For any modal logic $L$ between $K$ and $B$, the L-consistency relation is EXPTIME-hard.

Our method is borrowed and modified from that of Fischer and Ladner[5] for showing that the satisfiability problem of propositional dynamic logic (PDL) is EXPTIMEhard. The method is to reduce any language accepted by an alternating Turing machine operating in polynominal space to the $L$-consistency relation for any $L$ between $K$ and $B$. Since APSPACE = EXPTIME [1], the $L$-consistency relation thus is EXPTIMEhard. Similar methods have been used for showing EXPTIME-hardness of many intensional logics $[4,8,6]$.

Let $\mathcal{L}$ be any language accepted by an alternating Turing machine TM which operates in space $s(n)$, where $s$ is a polynominal,

Without loss of generality, assume that $s(n)$ is computable in time polynominal of $n$ and that any computation path of TM on any input never repeats a configuration. See Fischer and Ladner[5] for detail arguments.

Let $T M=\left(Q, \Gamma, \Sigma, \#, \delta, q_{0}, U\right)$ be a one-tape alternating Turing machine, where

- $Q$ is the set of states,

- $\Gamma$ is the tape alphabet,

- $\Sigma \subset \Gamma$ is the input alphabet,

- \# $\in \Gamma-\Sigma$ is the blank symbol,

- $\delta \subset(Q \times \Gamma) \times(Q \times \Gamma \times\{L, R\})$ is the next move relation,

- $U \subset Q$ is the set of universal states,

- $Q-U$ is the set of existential states, and

- $q_{0}$ is the initial state.

Let $x$ be any input string of length $n$. Assume $s(n)=m$. Since TM never runs outside the first $m$ tape cells, every configuration $x^{\prime} q y^{\prime}$ of TM possibly reachable from the initial configuration $q_{0} x$ can be represented by the triple $(q, i, z)$, where $i$ is the position (counted from 1 ) of the tape cell pointed to by the tape head and $z=\# x^{\prime} y^{\prime} \#^{\left(m+1-\left|x^{\prime}\right|-\left|y^{\prime}\right|\right)}$ is a string of length $m+2$ storing not only the nonblank part of the configuration but also all tailing blanks up to the $(m+1)$-th tape cell in the configuration; moreover, a blank is inserted at the front serving as end marker. Now define $C F^{m}$ to be the set of (representations of) all configurations of $T M$ of length at most $m$, i.e., $C F^{m}=\left\{(q, i, x)|q \in Q, 0 \leq i \leq m+1| x \mid, \in \# \Gamma^{m} \#\right\}$.

We construct a set of modal formulas $S_{T M(x)}$ using propositional variables from $P V_{T M(x)}$, which is given as follows.

1. $P V_{T M(x)}$ consists of the following propositional variables: 
- init, which is used to identify the initial configuration. init has the effect of preventing all predictions about successor configurations from being enforced to the initial configuration.

- $C_{i, a}$ where $0 \leq i \leq m+1$ and $a \in \Gamma$, which is used to represent the tape contents of current configuration so that $C_{i, a}$ is true iff the $i$ th tape cell contains $a$.

- $H_{i}$, where $0 \leq i \leq m+1$, which is used to represent the head position so that $H_{i}$ is ture iff the current tape head points to position $i$.

- $Q_{q}$, where $q \in Q$, which is used to represent the state of the current configuration.

- $L_{i}$, where $0 \leq i \leq 2$, which is used to represent the value $j \bmod 3$ where $j$ is the length of the computation path from the initial configuration to current configuration. $L_{i}$ has the effect of preventing all predictions about successor configurations from being enforced to predecessor configurations the current configuration.

2. $S_{T M(x)}$ is the union of the following sets.

- $S_{1}=\left\{\bigvee_{q \in Q}\left(Q_{q} \wedge \wedge_{q^{\prime} \in Q-\{q\}} \neg Q_{q^{\prime}}\right)\right\}$

$S_{1}$ is the state constraint which says that each configuration has exactly one state.

- $S_{2}=\bigcup_{0 \leq i \leq m+1}\left\{\bigvee_{a \in \Gamma}\left(C_{i, a} \wedge \wedge_{a^{\prime} \in \Gamma-\{a\}} \neg C_{i, a^{\prime}}\right)\right\} \cup\left\{C_{0, \#}, C_{m+1, \#}\right\}$

$S_{2}$ says that each tape cell contains a unique symbol in $\Gamma$ and the initial and the final cells must contain the blank symbol.

- $S_{3}=\left\{\neg H_{0}, \neg H_{m+1}\right\} \cup\left\{\bigvee_{1 \leq i \leq m}\left(H_{i} \wedge \wedge_{1 \leq j \leq m, j \neq i} \neg H_{j}\right)\right\}$

$S_{3}$ says that the tape head must point to exactly one position in $[1, m]$.

- $S_{4}=\left\{\neg H_{i} \wedge C_{i, a} \wedge L_{j} \supset \square\left(L_{j+1 \bmod 3} \wedge \neg i n i t \supset C_{i, a}\right) \mid 0 \leq i \leq m+1, a \in\right.$ $\Gamma, 0 \leq j \leq 2\}$

$S_{4}$ says that all tape cells not pointed to by tape head remain unchanged in the next configuration. Note that the $L_{j+1 \bmod 3} \wedge \neg i n i t$ appearing inside the box $\square$ has the effect of preventing the prediction that $C_{i, a}$ must be true from being enforced to the initial configuration, in which init must be true, and the current and the predecessor configurations in which $L_{j+1 \bmod 3}$ must be false.

- $S_{5}=\left\{H_{i} \wedge C_{i, a} \wedge Q_{q} \wedge L_{j} \supset \wedge_{\left(q, a, q^{\prime}, a^{\prime}, R\right) \in \delta} \diamond\left(H_{i+1} \wedge C_{i, a^{\prime}} \wedge Q_{q^{\prime}} \wedge L_{j+1 \bmod 3} \wedge\right.\right.$ $\neg$ init)^

$\bigwedge_{\left(q, a, q^{\prime}, a^{\prime}, L\right) \in \delta} \diamond\left(H_{i-1} \wedge C_{i, a^{\prime}} \wedge Q_{q^{\prime}} \wedge L_{j+1 \bmod 3} \wedge \neg\right.$ init $)$

$\mid 1 \leq i \leq m, a \in \Gamma, q \in U$ and $0 \leq j \leq 2\}$

$S_{4}$ and $S_{5}$ encode the next-move relation for universal states. It describes how the current configuration moves to successor configurations and says that 
the current configuration is accepted if all its successors are accepted. Note that the empty conjunction is defined to be T. The $L_{j+1 \bmod 3}$ 's appearing at the right hand side of the implication say that if the length modulo 3 of the path from the initial configuration to current configuration is $j$, then the length modulo 3 of the path from the initial configuration to every successor configuration is $j+1 \bmod 3$. The "ᄀinit"s appearing at the right hand side of the implication indicate that no successor configuration is the initial configuration.

- $S_{6}=\left\{H_{i} \wedge C_{i, a} \wedge Q_{q} \wedge L_{j} \supset \bigvee_{\left(q, a, q^{\prime}, a^{\prime}, R\right) \in \delta} \diamond\left(H_{i+1} \wedge C_{i, a^{\prime}} \wedge Q_{q^{\prime}} \wedge L_{j+1 \bmod 3} \wedge\right.\right.$ $\neg$ init $) \vee \bigvee_{\left(q, a, q^{\prime}, a^{\prime}, L\right) \in \delta} \diamond\left(H_{i-1} \wedge C_{i, a^{\prime}} \wedge Q_{q^{\prime}} \wedge L_{j+1 \bmod 3} \wedge \neg\right.$ ᄀinit $)$

$\mid 1 \leq i \leq m, a \in \Gamma, q \in Q-U$ and $0 \leq j \leq 2\}$

$S_{4}$ and $S_{6}$ encode the next-move relation for existential states. Note that the empty disjunction is defined to be 1 .

- Let $A_{T M(x)}$ denote the encoding of the initial configuration:

$Q_{q_{0}} \wedge H_{1} \wedge L_{0} \wedge C_{0, \#} \wedge \wedge_{1 \leq i \leq n} C_{i, x_{i}} \wedge \wedge_{n+1 \leq i \leq m+1} C_{i, \#}$.

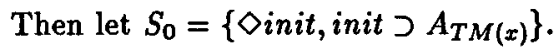

$S_{0}$ says firstly that there must exist a world at which init is true, and secondly that whenever init is true at a world, it is a world corresponding to the initial configuration. Note that $\diamond$ init has the side effect of making accessible from each world a world corresponding to the initial configuration.

The following proposition is easy to see.

Proposition $5 S_{T M(x)}$ can be obtained from (some encoding of) $T M$ and $x$ in polynominal time.

With the above explanation it is not hard to prove the following two lemmas, whose detail proofs can be found in Chen[3].

Lemma 6 [3] If $S_{T M(x)}$ is globally $K$-satisfiable, then $x$ is accepted by $T M$.

Lemma 7 [3] If $x$ is accepted by $T M$ then $S_{T M(x)}$ is globally B-satisfiable.

We can now prove the main theorem of this section.

Proof. (of Theorem 1.) Let $L$ be any modal logic between $K$ and $B$. Let $\mathcal{L}$ be any language in EXPTIME accepted by an alternating TM which operates in polynominal space $s(\cdot)$. Let $x$ be any input string. If $x$ is accepted by $T M$, then by Lemma 7 and Proposition 2, $S_{T M(x)}$ is globally B-consistent. Hence it is L-consistent. On the other hand, if $x$ is not accepted by TM, by Lemma 6 and Proposition $2, S_{T M(x)}$ is not $\mathrm{K}$-consistent. Hence it is not L-consistent. As a result, every language $\mathcal{L}$ in EXPTIME is polynominal-time reducible to the global L-consistency relation via the polynominal 
time transformation $(T M, x) \rightarrow S_{T M(x)}$. The L-consistency relation thus is EXPTIMEhard. $\bowtie$

Theorem 2 For $L \in\{K, T, B\}$, the $L$-consistency relation and the $L$-deducibility relation are EXPTIME-complete.

Proof. Direct consequence of Theorem 1 and Corollary 5 and the fact that EXPTIME is closed under complementation. $\bowtie$

\section{References}

[1] A.K. Chandra, D.C. Kozen and L.J. Stockmeyer, Alternation, J. of ACM 28,1981, 114-133.

[2] B.F. Chellas, Modal Logic: an Introduction, Cambridge university press, 1980.

[3] C.C. Chen, The complexity of decision problems for modal propositional logics, Ph.D. thesis, Department of Computer Science and Information Engineering, National Taiwan University. Taipei, Taiwan, 1993.

[4] E.A. Emerson and J.Y. Halpern, Decision procedures and expressiveness in the temporal logic of branching time, J. of Computer and System Science 30, 1985, $1-24$.

[5] M.J. Fischer and R.E. Ladner, Propositional dynamic logic of regular programs, J. of Computer and System Science 18, 1979, 194-211.

[6] M. J. Fischer and N. Immerman, Interpreting logics of knowledge in propositional dynamic logic with converse, Information Processing Letter 25, 1987, 175-181.

[7] M. Fitting, Proof Methods for Modal and Intuitionistic Logics, D. Reidel, 1983.

[8] J.Y. Halpern and Y.O. Moses, A guide to the modal logic of knowledge, Proc. of the 9th International Joint Conference on Artificial Intelligence (IJCAI-85), 1985, 480-490.

[9] D. Harel, Dynamic logic. in: D. Gabbay and F. Guenthner eds., Handbook of Philosophical Logic, vol 2: Extensions of Classical Logic, D. Reidel, 1984, 497604.

[10] G. E. Hughes and M. J. Cresswell, An Introduction to Modal Logic, Methuen \& Co., London, 1968.

[11] R.E. Ladner, The computational complexity of provability in systems of modal propositional logic, SIAM J. Comput. 6(3), 1977, 467-480.

[12] D. McDermott, Nonmonotonic logic II: nonmonotonic modal theories, J. of $A C M$ 29(1), 1982, 33-57.

[13] R.C. Moore, Semantical considerations on non-monotonic logic, Artificial Intelligence 25, 1985, 75-94. 
[14] G.F. Shvarts, Autoepistemic modal logics, In R. Parikh, ed., Proc. of the 3rd Conference on Theoretical Aspect of Reasoning about Knowledge, San Mateo, CA., Morgan Kaufmann, 1990, 97-109.

[15] M. Tiomkin and M. Kaminski, Nonmonotonic default modal logics, J. of $A C M$ 38, 1991, 963-984.

[16] H. Tuominen, Dynamic logic as a uniform framework for theorem proving in intensional logic, Proc. of the 10th International Conference on Automated Deduction, LNCS 449 Spring-verlag, Berlin, 1990, 514-527.

[17] E. Zarnecka-Bialy, A note on deduction theorem for Gödel's propositional calculus G4. Studia Logica 23, 1968, 35-40. 